\title{
Dobutamine Echocardiography for Assessment of Viability in the Current Era
}

Abhishek Khemka ${ }^{1}$, Stephen G Sawada ${ }^{1}$

Department of Cardiology, Indiana University School of Medicine/Indiana University Health, Indianapolis, Indiana, USA

Correspondence to Abhishek Khemka, MD, MBA

1800 N Capitol Avenue

Noyes Building, Suite E371

Indianapolis, IN 46202

Tel: +001 (317) 274-0992; Fax: +001 (317) 963-3340

Email: akhemka@iu.edu

Financial Disclosure: None

This is the author's manuscript of the article published in final edited form as:

Khemka, A., \& Sawada, S. G. (2019). Dobutamine echocardiography for assessment of viability in the current era. Current Opinion in Cardiology, 34(5), 484. https://doi.org/10.1097/HCO.0000000000000658 


\section{Purpose of Review}

Studies from the 1990s and early 2000s documented the utility of dobutamine echocardiography (DE) for prediction of functional recovery and prognosis with revascularization. The results of The Surgical Treatment of Ischemic Heart Failure (STICH) trial called into question the value of viability assessment using DE. The purpose of this review is to re-examine the literature on DE, put into context the STICH results, and provide insight into the current role of DE viability testing.

\section{Recent Findings}

In contrast to the results of previous nonrandomized trials, the STICH trial showed that patients with viability defined by nuclear perfusion imaging or DE did not have improved survival with CABG compared with optimal medical therapy. Viability by DE was defined as the presence of contractile reserve in $\geq 5$ segments with baseline dysfunction. The results of DE studies published before and after initiation of the STICH trial suggest that the definition of viability utilized in that trial may be suboptimal for assessment of improvement in global function and prognosis in patients undergoing revascularization. Assessment of global contractile reserve using wall motion score (WMS) or ejection fraction (EF) may be superior to utilization of a binary definition of viability confined to assessment of contractile reserve in a fixed number of segments because these indices provide information on both the magnitude and extent of contractile reserve of the entire left ventricle (LV).

\section{Summary}

Assessment of WMS or EF with DE may be the optimal means of evaluating the impact of viability on prognosis. (Supplementary Digital Content (SDC) call-out)

Key Words: Myocardial Viability, Dobutamine Echocardiography, Wall motion score 


\section{Key Points:}

- The STICH trial results suggested that viability testing may not be useful for outcome assessment but the trial had some limitations including how viability was defined.

- Defining viability based on a limited number of segments with contractile reserve may be insufficient for assessing prognosis and the benefit of revascularization.

- Viability assessment with dobutamine echocardiography should focus on evaluation of global contractile reserve using wall motion score or ejection fraction.

\section{Introduction:}

Dobutamine is a synthetic catecholamine with potent beta- 1 agonist effects originally developed as an intravenous agent for the acute treatment of congestive heart failure. ${ }^{1}$ At low doses, the agent improves stroke volume, lowers left atrial pressure and reduces systemic vascular resistance without causing substantial increases in heart rate and myocardial oxygen consumption. ${ }^{2}$ In the 1990 s, DE was developed as a method for assessment of viability in patients with chronic LV systolic dysfunction. The presence or absence of contractile response to dobutamine enables differentiation of nonviable tissue with transmural or extensive nontransmural infarction from stunned or hibernating myocardium. ${ }^{3,4}$ Hibernating myocardium may manifest small and transient increases in contractility at low doses of dobutamine followed by worsening of function with higher doses of dobutamine that increase heart rate and myocardial oxygen consumption. ${ }^{5}$ In the 1990s, animal and human studies showed that the demonstration of contractile reserve in dysfunctional myocardium correlated with the presence of viability by nuclear perfusion imaging and positron emission tomography using F-18 fluorodeoxyglucose. ${ }^{6,7}$

\section{Viability Assessment Before the Surgical Treatment of Ischemic Heart Failure (STICH) Trial}

In the 1990 s to early 2000 s, nonrandomized retrospective studies showed that in patients with ischemic LV dysfunction, revascularization of those with viable myocardium was associated with better outcomes compared to medical therapy. In a 2002 meta-analysis by Allman et al, the annual death rate was 3.2\% 
in subjects with viability who underwent revascularization compared to $16 \%$ in those with viability who were treated with medical therapy. The marked difference in survival was attributed both to improvement of ventricular function in those who were revascularized and an excess of ischemia related events in those treated medically. The meta-analysis also revealed that in patients without viability, revascularized and medically treated patients had similar annual mortality $\left(7.7 \%\right.$ and $6.2 \%$ respectively). ${ }^{8}$

\section{Defining Viability: The Controversy}

The Surgical Treatment of Ischemic Heart Failure (STICH) trial, which was initiated in 2002, randomized subjects who had ischemic ventricular dysfunction ( $\mathrm{EF} \leq 35 \%$ ) to coronary artery bypass grafting (CABG) or optimal medical therapy. The results of the viability substudy reported in 2011 , raised doubts about the utility of viability testing. ${ }^{9}$ In this study, patients were designated as having viability based on the number of segments with tracer activity on single photon emission computed tomography (SPECT), or $\geq 5$ dysfunctional segments exhibiting contractile reserve with dobutamine. Viability was not an independent predictor of outcome and patients with viability did not have improved outcome with CABG compared to medical therapy. The STICH definition of viability, based on contractile reserve in a fixed number of segments, was derived from some previous investigations showing that contractile reserve in approximately $25 \%$ of the LV was associated with modest increases ( $\geq 5 \%)$ in ejection fraction, improvement in functional class, and reduction in short term mortality with revascularization. ${ }^{10,11,12,13}$ Although contractile reserve in a fixed number of dysfunctional segments is a commonly accepted definition of viability, the limitations of a binary classification of patients as having viable or nonviable myocardium was pointed out in a 2002 editorial by Dr. Bonow. He stated that binary grouping, "oversimplifies the complex, inter-related continua of severity of LV dysfunction, extent and severity of inducible ischemia and magnitude of dysfunctional but viable myocardium.." 14

The limitations of a binary, 5 segment definition of viability was shown by Meluzin et al, who correlated the number of segments demonstrating contractile reserve with the degree of improvement in EF and outcomes in patients who underwent revascularization. In patients with 2 to 5 viable segments, 
revascularization improved EF by an average of $6 \%$ (34 to $40 \%$ ), but the rate of cardiac events after revascularization was similar to those with $<2$ viable segments. In contrast, in patients with $\geq 6$ viable segments, mean EF improved from $35 \%$ to $47 \%$ and cardiac events were significantly lower than in the other two groups. ${ }^{15}$ In a separate report, Meluzin et al also found a strong linear correlation $(r=0.91)$ between the number of segments with contractile reserve and post-revascularization improvement in EF suggesting that the degree of functional improvement and presumably outcome could be better assessed using a more continuous measure of viability. ${ }^{16}$ In a more recent investigation, Joshi et al, showed that contractile reserve in $25 \%$ of segments did not predict EF improvement after revascularization. ${ }^{17}$

\section{Ejection fraction and wall motion score for assessment of viability}

Assessment of the global contractile response to dobutamine using ejection fraction (EF) and wall motion score (WMS) are validated alternatives to reliance on a binary, 5 segment definition of viability. $18,19,20,21,22$ In a 1982 investigation, Nesto et al, reported that 5 year survival in patients with ischemic LV dysfunction was significantly better in revascularized patients with contractile reserve, defined as $\geq 10 \%$ augmentation of $\mathrm{EF}$, compared to those with lesser degrees of contractile reserve ( $80 \%$ vs $33 \%, p<0.01)$. Survival was also better in the medically treated group in the presence of $\geq$ $10 \%$ augmentation of EF $(75 \%$ vs $9 \%, p<0.01)$. Patients with $a \geq 10 \%$ augmentation of EF had a mean EF increase after revascularization of $17 \%$ compared with no improvement in EF in those with $<10 \%$ augmentation. Nesto, et al was the first to demonstrate that assessment of global contractile reserve can predict post-revascularization outcome, and that improvement in long-term survival might require substantially larger improvements in EF than a $5 \%$ increase. ${ }^{23}$ Joshi et al, showed that $\geq 8 \%$ improvement in EF with revascularization was an independent predictor of long-term survival. The $8 \%$ threshold was considered significant improvement in function on the basis of being twice the mean interobserver difference (3.7\%) in EF measurement. ${ }^{22}$

WMS is a well validated method for diagnostic and prognostic assessment of patients with ischemic dysfunction. 20,21,22,25 Visual analysis of wall motion and thickening is assessed in each segment of the left 
ventricle (16 or 17 segment model). Global WMS representing the average of individual segment scores can be derived at baseline and at various doses of dobutamine infusion. WMS incorporates information on both the severity and extent of segment dysfunction.

The utility of WMS as a non-dichotomous index of viability was initially reported in 2002. Low dose scores enabled further risk assessment of a mixed population of ischemic cardiomyopathy patients (revascularized and medically treated) initially risk stratified on the basis of the severity of baseline ventricular dysfunction. In patients with severely reduced systolic function (resting wall score $\geq 2.50$ ) the response to low dose dobutamine distinguished lower and higher risk groups (11 vs 30\% one year cardiac mortality). In patients with an intermediate extent of viability (low dose score 2.00 to 2.49), cardiac mortality was significantly lower in revascularized compared to medically treated subjects (7.5\% vs $20.5 \%){ }^{26}$

The value of low dose WMS for risk assessment of 95 patients who underwent surgical revascularization was assessed in a 2003 investigation. Patients had a mean EF of $33 \%$ by echocardiography, and $25 \%$ by contrast or nuclear angiography. Increasing low dose WMS was the most important predictor of cardiac death, and provided incremental prognostic value to clinical data and resting left ventricular function. The presence of contractile reserve in $\geq 4$ segments was not predictive of outcome. Survival progressively improved based on the extent of viability based on WMS. ${ }^{23}$

In ventricles with chronic dysfunction, myocardium that is normal, remodeled, nontransmurally injured, and transmurally infarcted coexists with stunned and hibernating tissue. The contractile response of all segments contribute to outcome since they mutually contribute to global ventricular function. Additionally, the extent of myocardium with transmural infarction contributes to prognosis and patients with extensive infarction may have poor outcomes in spite of revascularization. ${ }^{24,25}$ Figure 1 highlights a hypothetical example of the disparity that can occur in assessment of viability using contractile reserve in a limited number of segments versus wall motion score. Patient A has severe LV systolic dysfunction at 
rest by WMS with no normal segments and extensive nonviable (nonaugmenting) myocardium. However, the patient has 4 segments with contractile reserve and might be classified as having "viability" if defined on the basis of $25 \%$ of segments with contractile reserve. Patient B has less severe dysfunction based on resting WMS, and a large amount of normal myocardium along with a substantial region of transmural infarction. This patient would be classified as having "nonviabile" myocardium based on the absence of segments with contractile reserve. Apart from the influence of stress-induced ischemia on outcome, the long term prognosis of patient $B$ would be expected to be better than patient $A$, based on marked differences in the extent of normal and transmurally infarcted myocardium.

\section{Reevaluating viability testing after STICH}

Since the publication of the viability substudy results in 2011, there have been almost no further investigations employing DE for viability assessment. The dearth of recent investigations might imply that clinicians and investigators have accepted the STICH results as definitive and/or that the need for viability testing is declining because of expansion of alternative therapies such as mechanical assist devices. The viability substudy of STICH was acknowledged to have limitations (see table 1) beyond the echocardiographic definition of viability. The clinical impact of the viability substudy results rests on whether the STICH study population truly represented a population considered high risk for CABG. A number of characteristics suggest otherwise: although the intent was to enroll patients with $\mathrm{EF} \leq 35 \%$, EF could not be quantified by the core laboratory in $27 \%$ of patients and an additional $19 \%$ had EF measurements $>35 \%$. Furthermore, the annual mortality of $7 \%$ in medically treated patients with viability may be a reflection of the lower risk nature of the study population. ${ }^{14}$ A substantial proportion of patients enrolled in STICH, had moderate LV systolic dysfunction and extensive viable myocardium. In patients such as these, the impact of viability on prognosis may be outweighed by other factors including the extent and severity of coronary disease, and the presence and extent of ischemia.

\section{DE in the Contemporary Era}


The role of DE for assessment of viability needs to re-examined in light of the results of the STICH trial. EF and global WMS assessed during dobutamine infusion appear to be better measures of evaluating the prognostic impact of viability than assessment of contractile reserve in a limited number of segments. Viability testing is not needed in most patients with moderate LV dysfunction, angina and suitable anatomy for either surgical or percutaneous revascularization.

Low dose DE may remain a useful test in patients with severe global dysfunction, heart failure symptoms and multivessel disease who are candidates for revascularization. DE is a low cost modality that is widely available. ${ }^{26}$ In critically ill patients (i.e. on mechanical ventilation, with extracorporeal membrane oxygenation or with intra-aortic balloon pump support) that cannot be transferred out of the ICU, DE can be easily performed at the bedside in contrast to positron emission tomography or magnetic resonance imaging. Demonstration of global contractile reserve using low dose dobutamine with or without nitroglycerin may provide additional benefit for clinical decision making. ${ }^{27,28}$ Patients who demonstrate global contractile reserve may benefit from revascularization whereas patients that do not have substantial contractile reserve may be considered for other options such as mechanical assist device.

Low dose DE has also been shown to be a useful prognostic test in patients with LV dysfunction with $\mathrm{EF} \leq 35 \%$ and prolonged QRS $>120 \mathrm{~ms}$ who are candidates for cardiac resynchronization therapy (CRT). In a meta-analysis by Kloosterman et al, a positive response to DE defined by either an increase in LVEF or improvement in WMS was associated with a higher odds ratio for CRT response. This suggests that patients with demonstrable contractile reserve have viability and restoring synchrony improves overall function. ${ }^{29}$ In the ViaCRT study, investigators prospectively evaluated 127 patients with LVEF $\leq 35 \%$ on optimal pharmacotherapy and QRS $\geq 120 \mathrm{~ms}$. Contractile reserve with low dose DE was detected in $18 \%$ of patients by WMS and in $48 \%$ of patients by EF augmentation. At one year of follow-up after CRT, mortality was $4.4 \%$ in patients with contractile reserve by WMS versus $19.4 \%$ in those without contractile reserve. $^{30}$ 
DE has been investigated as a method to predict recovery after left ventricular assist device (LVAD) implantation and to guide explantation strategies with modest benefit. ${ }^{31,32}$ Khan et al, showed that in patients with LVAD who had a favorable response to DE $(n=9), 66 \%$ had successful explantation with no further hospitalizations and improvement in functional class NYHA I-II. ${ }^{31}$ DE may also be useful in predicting outcomes in patients with nonischemic cardiomyopathy. Wever-Pinzon et al evaluated contractile reserve using DE in HIV patients to predict EF improvement and found a change of WMS of 0.59 predicted improvement with a sensitivity of $74 \%$ and specificity of $78 \%$. ${ }^{33}$ McDermott et al explored the utility of using DE in 59 cancer patients with LVEF $<50 \%$ undergoing cardiotoxic chemotherapy to predict LV recovery and found $100 \%$ sensitivity and $85 \%$ specificity. ${ }^{34}$

\section{Conclusion:}

In contemporary practice, DE remains a useful diagnostic tool for assessment of viability in high risk candidates for revascularization and in other settings where determination of the extent and magnitude of contractile reserve can provide important information for clinical decision making. For evaluation of prognosis, global assessment of contractile reserve using wall motion score and ejection fraction appear superior to assessment of contractile reserve in a limited number of segments.

Acknowledgements: None. There are no financial disclosures. There are no conflicts to disclose.

\section{Supplemental Digital Content: Video Abstract}


${ }^{1}$ Akhtar N, Mikulic E, Cohn JN, Chaudhry MH. Hemodynamic effect of dobutamine in patients with severe heart failure. Am J Cardiol 1975; 36: 202-205.

${ }^{2}$ Ruffolo RR. Review: The Pharmacology of Dobutamine. Am J Med Sci 1987; 294: 244-248.

${ }^{3}$ Mertes H, Segar DS, Johnson M, et al. Assessment of hibernating myocardium by dobutamine stimulation in a canine model. J Am Coll Cardiol 1995; 26: 1348-1355.

${ }^{4}$ Gerber BL, Vanoverschelde JL, Bol A, et al. Myocardial blood flow, glucose uptake, and recruitment of inotropic reserve in chronic left ventricular ischemic dysfunction. Implications for the pathophysiology of chronic myocardial hibernation. Circulation 1996; 94: 651-659.

${ }^{5}$ Chen C, Li L, Prada JV, et al. Incremental doses of dobutamine induce a biphasic response in dysfunctional left ventricular regions subtending coronary stenoses. Circulation 1995; 92: 756-766.

${ }^{6}$ Baumgartner H, Porenta G, Lau YK, et al. Assessment of myocardial viability by dobutamine echocardiography positron emission tomography and thallium-201 SPECT. J Am Coll Cardiol 1998; 32: 1701-1708.

${ }^{7}$ Nagueh SF, Vaduganathan P, Ali N, et al. Identification of hibernating myocardium: Comparative accuracy of myocardial contrast echocardiography, rest-redistribution thallium-201 tomography and dobutamineechocardiography. J Am Coll Cardiol 1997; 29: 985-993.

${ }^{8}$ Allman KC, Shaw LJ, Hachamovitch R, Udelson JE. Myocardial viability testing and impact of revascularization on prognosis in patients with coronary artery disease and left ventricular dysfunction: a meta-analysis. J Am Coll Cardiol 2002; 39: 1151-8. (*)

${ }^{9}$ Bonow RO, Maurer G, Lee KL, et al. Myocardial viability and survival in ischemic left ventricular dysfunction. $\mathrm{N}$ Engl J Med 2011; 364: 1617-1625. (*)

${ }^{10}$ Bax JJ, Poldermans D, Elhendy A, et al. Improvement of left ventricular ejection fraction, heart failure symptoms and prognosis after revascularization in patients with chronic coronary artery disease and viable myocardium detected by dobutamine stress echocardiography. J Am Coll Cardiol 1999; 34: 163-9.

${ }^{11}$ Chaudhry FA, Tauke JT, Alessandrini RS, et al. Prognostic implications of myocardial contractile reserve in patients with coronary artery disease and left ventricular dysfunction. J Am Coll Cardiol 1999; 34: $730-738$.

${ }^{12}$ Senior R, Kaul S, Lahiri A. Myocardial viability on echocardiography predicts long-term survival after revascularization in patients with ischemic congestive heart failure. J Am Coll Cardiol 1999; 33: 1848-1854.

${ }^{13}$ Afridi I, Grayburn PA, Panza JA, et al. Myocardial viability during dobutamine echocardiography predicts survival in patients with coronary artery disease and severe left ventricular systolic dysfunction. J Am Coll Cardiol 1998; 32: 921-926.

${ }^{14}$ Bonow RO. Myocardial Viability and Prognosis in Patients with Ischemic Left Ventricular Dysfunction. J Am Coll Cardiol 2002; 39: 1159-1162. (*)

${ }^{15}$ Meluzin J, Cerny J, Frelich M, et al. Prognostic value of the amount of dysfunctional but viable myocardium in revascularized patients with coronary artery disease and left ventricular dysfunction. Investigators of this Multicenter Study. J Am Coll Cardiol 1998; 32: 912-20.

${ }^{16}$ Meluzin J, Cigarroa CG, Brickner ME, et al. Dobutamine Echocardiography in predicting improvement in global left ventricular systolic function after coronary bypass or angioplasty in patients with healed myocardial infarcts. Am J Cardiol 1995; 76: 877-880.

17 Joshi K, Alam I, Ruden E, et al. Effect of improvement in left ventricular ejection fraction on long-term survival in revascularized patients with ischaemic left ventricular systolic dysfunction. Eur J Echocardiogr 2011; 12 : $454-60$.

${ }_{18}$ Nesto RW, Cohn LH, Collins JJ, et al. Inotropic contractile reserve: A useful predictor of increased 5 year survival and improved postoperative left ventricular function in patients with coronary artery disease and reduced ejection fraction. Am J Cardiol 1982; 50: 39-44.

${ }^{19}$ Cain P, Khoury V, Short L, Marwick TH. Usefulness of quantitative echocardiographic techniques to predict recovery of regional and global left ventricular function after acute myocardial infarction. Am J Cardiol 2003; 91: 391-396.

${ }^{20}$ Moller JE, Hillis GS, Oh JK, et al. Wall motion score index and ejection fraction for risk stratification after acute myocardial infarction. Am Heart J 2006; 151: 419-425.

${ }^{21}$ Sawada SG, Lewis SJ, Foltz J, et al. Usefulness of rest and low-dose dobutamine wall motion scores in predicting survival and benefit from revascularization in patients with ischemic cardiomyopathy. Am J Cardiol 2002; 89: 811816. 
${ }^{22}$ Sawada SG, Dasgupta S, Nguyen J, et al. Effect of revascularization on long-term survival in patients with ischemic left ventricular dysfunction and a wide range of viability. Am J Cardiol 2010; 106(2): 187-92.

${ }^{23}$ Sawada S, Bapat A, Vaz D, et al. Incremental value of myocardial viability for prediction of long-term prognosis in surgically revascularized patients with left ventricular dysfunction. J Am Coll Cardiol 2003; 42: 1999-2105.

${ }^{24} \mathrm{Kwon} \mathrm{DH}$, Halley CM, Carrigan TP, et al. Extent of left ventricular scar predicts outcomes in ischemic cardiomyopathy patients with significantly reduced systolic function: a delayed hyperenhancement cardiac magnetic resonance study. J Am Coll Cardiol: Cardiovasc Imaging. 2009; 2: 34-44.

${ }^{25}$ Liu Y, Jiang Y, Yang X, et al. Limited prognostic value of myocardial viability assessment in patients with coronary artery diseases and severe left ventricular dysfunction. J Thorac Dis 2018; 10: 2249-2255.

${ }^{26}$ Patel H, Mazur W, Williams KA, et al. Myocardial Viability-State of the art: Is it still relevant and how to best assess it with imaging. Trends Cardiovasc Med 2018; 28: 24-37.

${ }^{27} \mathrm{Ma} \mathrm{L}$, Chen L, Gillam L, et al. Nitroglycerin enhances the ability of dobutamine stress echocardiography to detect hibernating myocardium. Circulation 1997; 96: 3992-4001.

${ }^{28}$ Ling LH, Christian TF, Mulvagh SL, et al. Determining myocardial viability in chronic ischemic left ventricular dysfunction: a prospective comparison of rest-redistribution thallium 201 single-photon emission computed tomography, nitroglycerin-dobutamine echocardiography, and intracoronary myocardial contrast echocardiography. Am Heart J 2006; 151: 882-889.

${ }^{29}$ Kloosterman M, Damman K, Van Veldhuisen DJ, et al. The importance of myocardial contractile reserve in predicting response to cardiac resynchronization therapy. Eur J Heart Fail 2016; 19: 862-869.

${ }^{30}$ Plonska-Gosciniak E, Kasprzak JD, Kukulski T, et al. Role of low-dose dobutamine echocardiography in predicting response to biventricular pacing. Results from the multicenter Viability in Cardiac Resynchronisation Therapy (ViaCRT) study. Pol Arch Med Wewn 2016; 126: 989-994.

${ }^{31}$ Khan T, Delgado RM, Radovancevic B, et al. Dobutamine stress echocardiography predicts myocardial improvement in patients supported by left ventricular assist devices (LVADs): hemodynamic and histologic evidence of improvement before LVAD explantation. J Heart Lung Transplant. 2003; 22: 137-146.

32 Maybaum S, Mancini D, Xydas S, et al. Cardiac Improvement during mechanical circulatory support: A prospective multicenter study of the LVAD working group. Circulation 2007; 115: 2497-2505.

${ }^{33}$ Wever-Pinzon O, Bangalore S, Romero J, et al. Inotropic Contractile Reserve Can Risk-Stratify Patients with HIV Cardiomyopathy. J Am Coll Cardiol: Cardiovasc Imaging 2011; 4: 1232-1238.

${ }^{34}$ McDermott L, Laeeq R, Palaskas N, et al. Using Dobutamine Stress Echocardiography to Predict the Recovery of Left Ventricular Function in Cancer Patients Receiving Chemotherapy. J Am Coll Cardiol 2019; 73: 1604. Abstract. 